\section{Relation between speed of learning and rate of forgetting in the elderly}

\author{
JAMES C. DIXON, University of Florida, \\ Gainesville, Fla. 32601
}

The question whether or not faster elderly learners are slower forgetters, when all learn to some criterion, was tested by a paired-associates learning, recall, and relearning task. The learning criterion was two successive correct responses to each stimulus word learned by the anticipation method. Ss were 46 guests of nursing and retirement homes between the ages of 65 and 80. It was found that the slower elderly learners showed poorer retention over a 15-min interval, as measured by recall and relearning.

The question whether or not faster learners are slower forgetters used to be answered in the affirmative (e.g., Gillete, 1936); this suggested a functional dependency of at least some learning and retention processes. In 1954, however, Underwood reversed his own earlier affirmative position (1949) and now (1954) contends that the rate of retention is essentially independent of learning rate. He believes that the reported poorer recall of slow learners is generally a matter of not learning as well in the first place.

In the case of the mentally impaired elderly, where discrepancy between memory for recent and remote events is often noted, it is quite likely that recent events are not attended to and rehearsed as well as remote events were in earlier years. This no doubt accounts for some of the poorer memory of the elderly, but the failure of recall of even well learned material, along with decline in learning facility, is easily observed in the mentally impaired elderly. Therefore, while learning speed and forgetting may be largely independent in the unimpaired young (Stroud \& Carter, 1961), it is possible that they may become increasingly related in the decline of old age.

This study reports learning and recall data on a paired-associates task given to elderly $\mathrm{Ss}$ who varied considerably in degree of mental decline. To the extent that both learning and retention processes are similar or similarly affected, the slower elderly learner should show relatively poorer retention than the faster learner, even though he learns to the same initial criterion level. Thus, a negative relation should obtain between number of trials required to learn and amount later recalled, rather than a zero order relation that might be expected if learning and recall were functionally independent. $S$ 's residence.

\section{SUBJECTS} to the learning and recall task.

\section{RESULTS} level.
Table 1

Learning and Relearning Errors on Hard Pairs Compared with Recall Score Level

\begin{tabular}{|c|c|c|c|c|c|}
\hline \multirow{2}{*}{$\begin{array}{l}\text { Recall } \\
\text { Score }\end{array}$} & \multirow{2}{*}{$\mathbf{N}$} & \multicolumn{2}{|c|}{ Learning } & \multicolumn{2}{|c|}{ Relearning } \\
\hline & & $\mathbf{M}$ & $\sigma$ & $\mathbf{M}$ & $\sigma$ \\
\hline 3 & 10 & 2.80 & 2.14 & 0.30 & .64 \\
\hline 2 & 13 & 5.85 & 4.78 & 1.39 & .83 \\
\hline 1 & 11 & 5.64 & 3.42 & 3.82 & 1.85 \\
\hline 0 & 12 & 10.42 & 5.02 & 7.08 & 3.78 \\
\hline
\end{tabular}

Each $S$ individually learned seven word pairs from Inglis (1959) by the method of anticipation. There were four easy pairs (hand-foot, knife-fork, cat-milk, gold-lead) and three hard pairs (table-river, flower-spark, bottle-comb). The easy and hard pairs were alternated, and the list was read at the rate of one pair every $2 \mathrm{sec}$. Then, after a 5-sec interval, the first word of each pair was read in a random order, allowing about $5 \mathrm{sec}$ for a response. The list was then read again, followed by presenting each stimulus word as before and requesting anticipatory recall of the correct associate. A word pair was discontinued after two successive correct responses. Ss were not advised that later recall would be requested. An interval of $15 \mathrm{~min}$ between learning and recall was filled with such unrelated tasks as mental arithmetic and color naming. Following the recall test, by the identical method of anticipation, the word pairs were then relearned. The entire task was completed at one session in a private room at

Guests in nursing and retirement homes, between the ages of 65 and 80 , volunteered for testing in connection with a study of mental impairment. There were 46 Ss out of 72 who achieved both a learning and relearning criterion of two successive correct repetitions of each pair within a maximum of 42 total repetitions or 10 trials for any one pair. Few of the more severely impaired were able to complete the task under the limiting conditions that were imposed to reduce frustration. There were 21 men and 25 women. Mean age was $74 \pm 4$ years. Neither age nor sex was significantly related

The correlation between number of trials to learn and the number of associates recalled after $15 \mathrm{~min}$ was -.56 , and that between trials to learn and trials to relearn was .64 , while relearning and recall correlated -.88 . All these product-moment coefficients are significant beyond the .01

The relation between learning and relearning shows that the more efficient learner also relearns more quickly, while the relation between learning and recall (and that between recall and relearning) suggests that he releams more quickly because he retains more of the original learning during the interval. This is shown in Table 1 , where learning and relearning errors on the hard pairs are compared with recall score level. It may be seen that those who recalled more required fewer trials to learn and made fewer relearning errors, both absolutely and relative to original learning score. (Analysis of the easy pairs is not included because of restricted variance among the fast learners, and also because the retention time is notably longer for the slower learners since the easier pairs were learned relatively more quickly and dropped out earlier.)

\section{DISCUSSION}

If it be granted that the learning criterion of two successive correct repetitions of each of the three hard word pairs represents an approximately similar level of initial learning for the slower and faster learners, then retention in the elderly can hardly be considered to be independent of the course of learning.

The question of whether or not the slower learners did, in fact, reach the same learning level at the end of the learning phase presents a knotty criterion problem complicated by the method of anticipation.

If a distinction between learning and recall is made in terms of length of retention interval, it is clear that the operations are similar for determining either amount learned or amount recalled. The only difference is the length of the retention interval, a few seconds, or hours, or days before requesting recall. Given a fixed criterion of two successive correct responses for purposes of defining equal degrees of original learning, the problem is one of determining rates of forgetting by faster or slower learners. On the other hand, equal degrees of original leaming can be defined in terms of number of correct repetitions required to achieve a certain level of recall after a stated interval. In either case, it appears that the slower learner- (faster forgetter?) requires a greater number of input units per unit output per unit time.

\section{REFERENCES}

GILLETTE, A. L. Learning and retention: A comparison of three experimental procedures. Archives of Psychology, N.Y., 1936, 28, No. 198.

INGLIS, J. A paired-associate learning test for use with elderly psychiatric patients. Journal of Mental Science, 1959, 105, 440-443.

STROUD, J. B., \& CARTER, L. J. Inhibition phenomena in fast and slow learners. Journal of 
Educational Psychology, 1961, 52, 30-34.

UNDERWOOD, B. J. Experimental psychology: An introduction. New York: Appleton-Century-Crofts, 1949.

UNDERWOOD, B. J. Speed of learning and amount retained: $A$ consideration of methodology. Psychological Bulletin, 1954, 51, 276-282.

UNDERWOOD, B. J. Forgetting. Scientific American, 1964, 210,91-99.

\section{Reminiscence in recognition memory for faces}

GRAEME WALLACE, MAX COLTHEART, ${ }^{1}$ and KENNETH $I$. FORSTER, Monash University, Clayton, Victoria, Australia

Milner (1968) demonstrated the occurrence of reminiscence in recognition memory for photographs of faces. The existence of this effect was confirmed, and the effects of inspection time and of varying the inspection-test delay were studied. The results were considered in the context of the work of Kleinsmith \& Kaplan (1963) on arousal and memory.

Underwood (1953, p. 47) noted that reminiscence could virtually be described as a "prewar" phenomenon and this state of affairs has prevailed until quite recently. However, a resurgence of interest in this phenomenon has recently occurred (Hochberg \& Galper, 1967; Scheirer \& Voss, 1969). One aspect of the problem has been dealt with by Milner (1968), who demonstrated a reminiscence effect in recognition memory for faces. In this experiment, Ss viewed an inspection series of 12 photographs of faces for $45 \mathrm{sec}$ and were then presented with a test series of 25 such photographs and instructed to indicate which 12 of these they had seen in the inspection series. Recognition accuracy was significantly greater when there was a 90-sec delay between inspection and test than when there was no delay.

The aim of the experiments reported here was to explore this effect by varying both the inspection time and the delay between inspection and test. METHOD

The Ss were 200 trainee teachers enrolled at a Teachers' College. They were all unpaid volunteers and were tested in groups of four. Each $S$ viewed an inspection series of 12 faces and then a test series of 25 faces, and he was told to choose the 12 he had previously seen. The 25 photographs were full-face, head-and-shoulder photographs of students from another teaching institution. They were $2 \times 2 \frac{1}{4}$ in. and were mounted in a 5 by 5 array on a sheet of cardboard; the test series was similarly mounted in a 4 by 3 array. The test series combined photographs of 12 men and 13 women; the inspection series contained photographs of 6 men and 6 women.

Half the Ss viewed the inspection series for $45 \mathrm{sec}$; half viewed it for $15 \mathrm{sec}$. Within each of these two groups, five different inspection-test delays were used: $0,45,90$, 180 , and $360 \mathrm{sec}$. Thus, there were 10 groups, each of 20 Ss.

\section{RESULTS}

The mean number of faces correctly recognized (out of 12) is plotted in Fig. 1 as a function of delay interval for each inspection period. The means and variances of each group are given in Table 1.

Analysis of variance revealed a significant effect of delay time. $[F(4,190)=3.20, p<.02$. The functions relating mean number recognized to delay period were highly similar for the two inspection periods, with a rise from 0 to $45 \mathrm{sec}$, a decline, and then a slight increase to $360 \mathrm{sec}$. Mann-Whitney U tests showed that recognition was significantly better at $45 \mathrm{sec}$ delay than at $0 \mathrm{sec}$ delay for the 45 -sec inspection group $(p<.05)$. For the 15 -sec inspection group, this effect did not reach significance, because it was obscured by a ceiling effect. That is, a considerable number of Ss achieved the maximum score (12) at each delay condition.

The same experiment was conducted

Table 1

Mean Scores for Groups of 20 Ss at Various Delays and Presentation Rates Delay Between Inspection and Retest (in Sec)

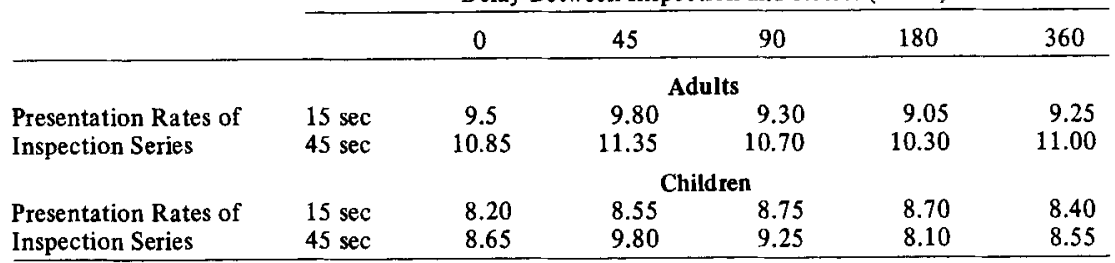
performance reflects the adequacy of using 200 children, aged 10 to 12 years, as Ss. The results are given in Fig. 2. Neither the effects of delay $[F(4,190)=2.20$, $p>.05]$ nor the effects of inspection period $[F(1,190)=2.27, p>.05]$ reached significance. However, for both inspection periods, performance was better at $45 \mathrm{sec}$ delay than at $0 \mathrm{sec}$ delay. The function relating recognition accuracy to delay with 45-sec inspection period was very similar to the two adult functions shown in Fig. 1. The 15-sec function exhibited some differences, possibly because this period was too short for the children to cope with.

\section{DISCUSSION}

Although a ceiling effect presented difficulties in data analysis, clear evidence for a reminiscence effect in recognition memory for faces was obtained. This effect occurred at a 45-sec inspection-test delay rather than at the $90-\mathrm{sec}$ delay as found by Milner (1968).

The demonstration of reminiscence effect for recognition memory raises problems for the view that recall and recognition are fundamentally different processes. Kintsch (1968) has suggested that retrieval is involved in recall but not in recognition. Hence, organization of the stimulus material, which is thought to affect retrieval, exerts no effect on recognition. By implication, recognition initial storage more directly than does recall. But to account for reminiscence effects, it would have to be assumed that the actual storage of the information improves in some way during the delay interval. Alternatively, it can be assumed that retrieval is also involved in recognition (Mandler et al, 1969), and that it is the efficiency of the retrieval mechanism that is affected by the delay.

A speculative answer to the question concerning the cause of this effect is provided by the work of Kleinsmith \& Kaplan (1963), who found that material that produced high arousal was less well recalled than low-arousal material, with immediate recall. However, the material was better recalled than was low-arousal material when a delay was introduced between presentation and recall. McLean 